\title{
The Concept of space-time quanta in future technologies
}

\author{
Igor Maydykovskiy, Petras Užpelkis
}

«Biveja» JSC, Private Research Laboratory (Lithuania)

www.biveja.com e-mail: biveja@gmail.com

\begin{abstract}
The article discusses the possibility of using the technological advantages that appear in connection with the discovery of the physical essence of Time and new interpretation of the structure of space in the form of space-time quanta. One of the problems that can be successfully solved on the basis of the new physical model is the problem of establishing the true nature of gravity. The solution to this problem is directly related to the implementation of the idea of unsupported motion based on the interaction in a certain way of the created asymmetric interference structure of longitudinal waves with the natural structure of spatial frequencies.
\end{abstract}

Key words: Space-frequency-time continuum, Fifth dimension, physical essence of Time, constant of fine structure, evolutionary spiral, nature of gravity.

\section{Introduction}

The holographic principle of organizing the structure of Space and the fractal principle of energy distribution with a discrete nature of the change in the states of a material object made it possible to formulate the Postulate of nested sub-spaces [1]. According to this postulate, space is discrete and consists of a set of equal wave matrices. The formation and division of the quantum space into subspaces occurs according to the frequency principle: one copy-embodiment frequency, - one wave matrix, - one frequency horizon, - one space-time quantum. Accordingly, the 5 th dimension in the coordinates of the space-frequency-time continuum: $\left(\mathrm{X}, \mathrm{Y}, \mathrm{Z}, \mathrm{T}, \mathrm{H}_{\mathrm{F}}\right)$ is represented by a twocoordinate frequency scale. One coordinate of this scale represents the overall scale of all frequencies $\left(\tilde{v}_{H G}\right)$, and the other coordinate represents the scale of the spectral bandpass filters $\tilde{\boldsymbol{v}}\left(H_{\boldsymbol{F}}\right)$. Each spectral range of this scale corresponds to its own sequence of harmonic series, which, in comparison with the rest of the ranges, is based on mutually exclusive frequencies.

Also in this work, in the form of a scientific Discovery, a previously unknown definition of such a concept as Time is presented. The formula of scientific Discovery: The physical essence of Time is manifested as a fraction of electromagnetic energy spent on updating a material object in a cyclic process of copying - incarnation. The value of this fraction, presented as the tangent of the loss angle, is a fundamental constant, identically equal to the square of the fine structure constant $\left(\boldsymbol{\alpha}^{2}\right)$ and, being represented in radians, determines the "tilt angle" of the evolutionary spiral, thereby regulating the period of state change, the duration of events or processes.

\section{About the True Nature of Gravity}

One of the main problems that can be successfully solved using the theory of a phase medium is the problem of establishing the true nature of gravity, which since Newton's time has actually remained a hostage of empirical formulas and unfounded conclusions regarding the propagation of so-called gravitational waves invented by Einstein.

To be convinced of the erroneousness of the theory of gravity in relation to planetary scales, it is enough to recall Kepler's third law, which establishes one of the basic harmonic laws that links the period of a planet's revolution with the radius of its orbit. 
In an accessible form, this law can be represented as follows, - If the distance $(R)$ from the planet to the Sun is raised to a cube, and then the result is divided by the period (T) raised to a squared, then a certain quantity will be obtained. For all planets of the solar system, the value of this quantity turns out to be a constant:

$$
\frac{\left(R_{1}\right)^{3}}{\left(T_{1}\right)^{2}}=\frac{\left(R_{2}\right)^{3}}{\left(T_{2}\right)^{2}}=\text { Const; }
$$

With the advent of Newton's "law of universal gravitation", the scientific community did not remember the harmonic principle incorporated into Kepler's law. Newton connected this constant with the mass of the Sun, considering that the constant is nothing more than a certain gravitational potential of the Sun or, through a conversion factor, simply its mass. Therefore, the gravitational constant $(\mathrm{G})$ was introduced into the "conversion" factor. As a result, the formula was presented in the following form:

$$
\frac{4 \pi^{2}}{G} \cdot \frac{R^{3}}{T^{2}}=\text { Mass of the Sun }(\mathrm{kG}) \text {; }
$$

Thus, after Newton artificially introduced the gravitational constant $(G)$ into the formula of Kepler's law, the formula itself turned into an empirical dependence, giving the gravitational constant the appearance of the necessity of its existence.

In fact, Kepler's constant relates the energy of the angular momentum to the frequency of the planet's revolution around the parent body, that is, it is a typical wave constant for the solar planetary system. As you know, a standing spherical longitudinal wave is characterized by the constancy of potential energy, which is concentrated at the nodes of the standing wave and cannot be transferred lengthways the radius of the sphere. The magnitude of this energy can be defined as a quantum of energy, then Kepler's law will reflect the fact that the magnitude of the quantum of energy is constant for all orbital channels within the planetary system.

Thus, in each of the nodes of the spherical standing wave, a potential well is formed in the form of an orbital channel, thereby determining the position of each planet lengthways the radius of the sphere of the solar's frequency funnel. Accordingly, the orbital parameters of the planet, according to Kepler's law, will be determined only by the potential energy concentrated at the node of the standing wave, which means that they will not depend on the mass of the planet and on non-existent gravity.

The result of ignoring Kepler's third law was the fact that the diameters of the orbits of the planets of the solar system are compared by means of an empirical relationship in accordance with the TitiusBode rule. Although it is obvious that, taking into account the presented physical model, the law of the location of orbital channels lengthways the radius of the sphere of the frequency funnel obeys the wave equation: $\quad \Delta^{2} \xi-\frac{1}{v^{2}(r)} \cdot \frac{\partial^{2} \xi}{\partial t}=0$;

here, $\boldsymbol{\Delta}$ - Laplace operator characterizes the density of the potential vector field.

The solution of the wave equation in a spherical coordinate system for a standing longitudinal spherical wave in an elastic medium will have the form:

$$
\boldsymbol{\varphi}=\frac{1}{r} \cdot \varphi_{1}[r-t \cdot v(r)]+\frac{1}{r} \cdot \varphi_{2}[r+t \cdot v(r)] ;
$$

here, $\varphi_{1}$ - is a spherical wave diverging from the origin of coordinates;

$\boldsymbol{\varphi}_{2}$ - spherical wave converging to the origin;

$\boldsymbol{v}(\boldsymbol{r})$ - is the functional dependence of the phase velocity on the radius.

It is obvious that calculating the value of the orbital radius for each of the planets is not an easy task. 
If we take into account that the value of the phase velocity within of the sphere of the solar's frequency funnel depends on the density of the medium, which in turn, according to the law of a power function, functionally depends on the radius [3]. However, there is no doubt that the result of solving this equation will fully comply with Kepler's third law and will convincingly confirm the correctness of the presented model.

The diagram, Fig. 1 shows the arrangement of the planets of the solar system at the nodes of a spherical standing longitudinal wave, corresponding to the proposed physical model. Here the existence of a frequency funnel is indicated not by the density of the medium, but by the magnitude of the density gradient: grad $\vec{\rho}$, which makes it possible to display the change in potential energy inside the sphere of the frequency funnel on a linear scale.

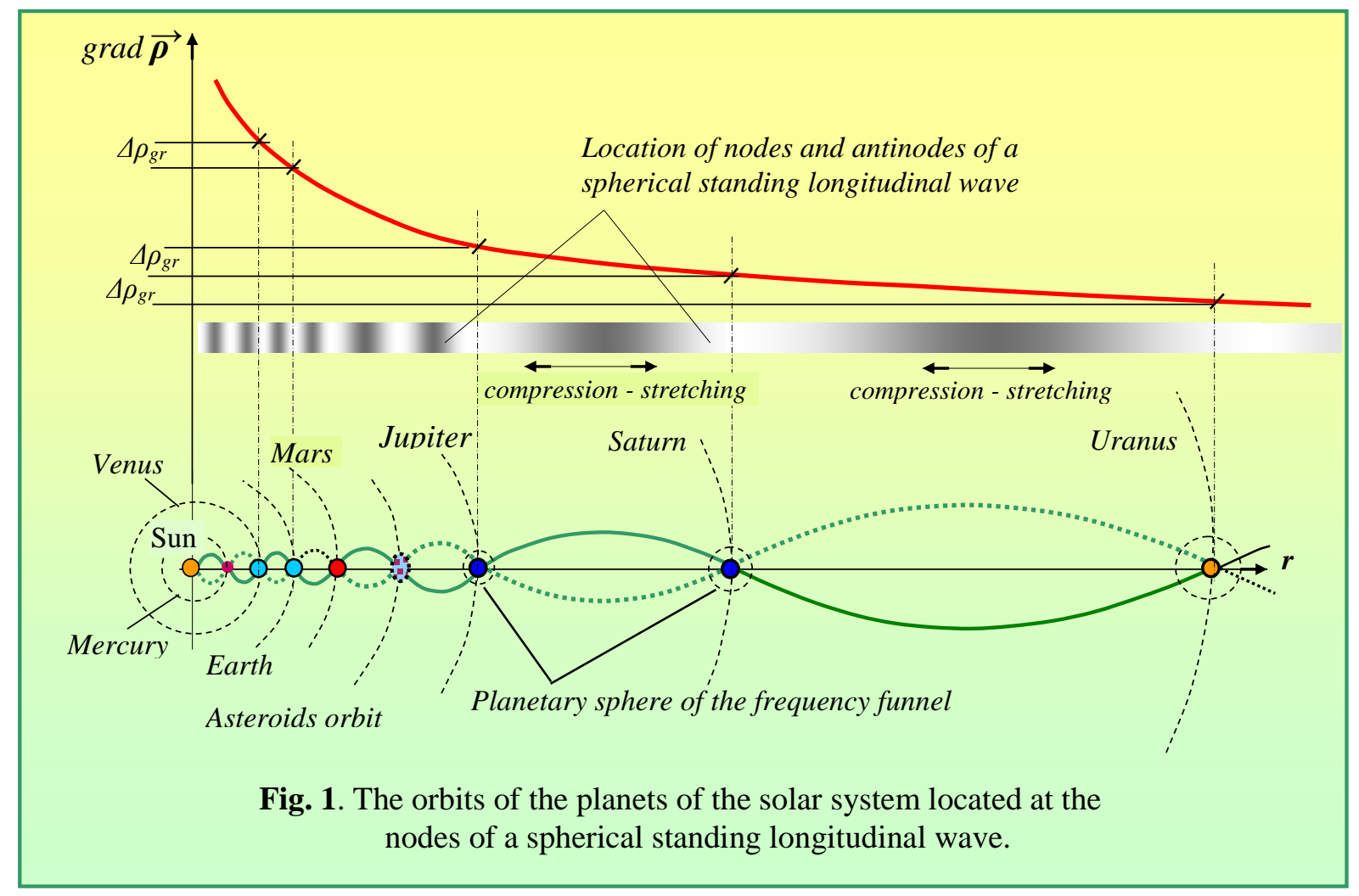

Then, reflecting the physical essence of Kepler's law, the magnitude of the energy quantum will be proportional to the difference in the density gradients of the medium of two adjacent orbital channels:

$$
\Delta \boldsymbol{\rho}_{\operatorname{gr}}=\operatorname{grad} \overrightarrow{\boldsymbol{\rho} \mathbf{1}}-\operatorname{grad} \overrightarrow{\boldsymbol{\rho} \mathbf{2}}=\operatorname{grad} \overrightarrow{\boldsymbol{\rho} \mathbf{2}}-\operatorname{grad} \overrightarrow{\boldsymbol{\rho}}=\text { Const }
$$

A more detailed analysis of the results obtained will be performed in a separate article devoted to planetary theory. Here we present some of the results of already carried out studies that relate to the structure of spatial frequencies within the sphere of the earth's frequency funnel.

According to the physical model, the boundary of the sphere of the earth's frequency funnel is determined by the libration point (equilibrium point). This distance corresponds to the radius of the sphere: $\boldsymbol{r}_{L}=1500 \cdot 10^{3}(\mathrm{~km})$. Obviously, within the framework of the planetary theory without gravity, the term "equilibrium" cannot be used, since the boundary of the sphere is characterized by an abrupt change in the gradient of the density of the medium. Knowing the mean radius of the Moon's orbit: $\boldsymbol{r}_{\boldsymbol{m}}=384 \cdot 10^{3}(\mathrm{~km})$, it can be argued that in accordance with the physical mechanism of formation of the structure of a spherical standing wave and according to Kepler's third law (formula 14-1), within the sphere of the earth's frequency funnel, in addition to the lunar orbit, at least one more additional orbital channel must exist. 
According to the physical model of the hierarchical structure of matter [2], the dependence of the density gradient of the medium on the radius of the spherical shell in general form can be expressed through the phase gradient of the spatial frequency:

$$
\operatorname{grad} \overrightarrow{\boldsymbol{\rho}}(\boldsymbol{r})=\operatorname{grad} \boldsymbol{\varphi}=\frac{\partial}{\partial t} \frac{\partial v}{\partial \vec{r}} ;
$$

The existence of the Earth's frequency funnel was confirmed by Harvard University researchers Robert Pound and Glen Rebka in 1960. Although, for obvious reasons, they themselves did not know about it, when in a laboratory controlled experiment they decided to check the "slowing down of time" in the Earth's gravitational field [4]. It is believed that the experiment confirmed the existence of a gravitational "red-shift". To determine the supposedly "difference in the rate of time flow" at the points spaced apart in height, Pound and Rebka used the measurement of the photon frequency, $\boldsymbol{v}=$ $2.2 \cdot 10^{19}(\mathrm{~Hz})$ at two points lengthways their trajectory - at the point of emission and at the point of absorption. A photon emitted by a $F e^{57}$ nucleus in a transition with an energy of $14.4 \mathrm{keV}$ traveled a distance $h=22.5(\mathrm{~m})$ vertically in the Earth's gravitational field and was resonantly absorbed by a target made of the same material. The measured difference in the value of frequencies at the upper and lower points, allegedly indicated the difference in the course of time at these points.

To understand the erroneous interpretation of the experimental results, it is necessary to explain the experimental results without involving gravity and the theory of relativity. As the researchers themselves note, the characteristics of the samples of the source and the radiation detector were identical. In other words, in their opinion, the resonance properties of the $\mathrm{Fe}^{57}$ crystal lattice remained unchanged and did not depend on the distance to the Earth's surface.

In fact, the dimensions of the crystal lattice, and hence the natural frequency, change under the influence of the density gradient of the medium. That is, against the background of an increase in the natural frequency of the atomic clock located at the surface of the Earth, it will seem as if the frequency measured by the detector, during the resonant absorption of photons coming from the emitter installed at an altitude, is shifted on the spectrogram to the low frequency region. Which will be perceived as "red-shift" or as time dilation. Accordingly, in the opposite situation, the detector installed above the emitter will register an increase in frequency against the background of its own lower-frequency oscillations. The paradox of the situation lies in the fact that it is not possible to use a second analogous atomic clock for this purpose, since for this it is necessary to compare not the frequencies, but the difference between the number of quantum transitions separately for the source and separately for the detector.

When analyzing the Sagnac effect [5], a technique was demonstrated that allows one to interpret the number of quantum transitions as the number of photon incarnations expressed in pixels $\left(P_{\text {inc }}\right)$. In fact, the physical mechanism of the formation of a frequency funnel creates conditions for the propagation of photons in a phase medium similar to the conditions that arise in a ring resonator when a Sagnac interferometer rotates. With the difference that the difference in the number of incarnations $\left(\Delta P_{i n c}\right)$ of two counterpropagating waves is associated not with the mechanical movement of the resonator, but with different conditions of the state of the phase medium. When two interferometers are placed at different heights, the difference in the number of incarnations $\left(\Delta P_{\text {inc }}\right)$ appears in the structure of the potential field as a result of changes in the gradient of the density of the medium. This difference can be determined from the number of quantum transitions during the propagation of rays in each of the two interferometers.

For applied purposes, using atomic clocks with an accuracy of $10^{-16}(\mathrm{sec})$, such measurements have repeatedly confirmed the dependence of the resonance frequency of atomic vibrations on the height. 
Returning to the experiment of Pound and Rebka, we can state that this experiment clearly proves that the change in the resonant frequency of photons, allegedly associated with the difference in gravitational potentials and allegedly leading to a slowdown in the velocity of photons, is in fact $\mathbf{a}$ consequence of the deformation of the crystal lattice of $\mathrm{Fe}^{57}$ crystals and the atoms themselves under by the influence of the frequency gradient, which occurs in the structure of spherical longitudinal waves within the sphere of the earth's frequency funnel.

It is obvious that the frequency-phase gradient of the external field will deform the internal interference field of any object located at the Earth's surface. At the same time, in the process of deformation, the zone of energy comfort of the object will tend towards the center of the frequency funnel, that is, towards the center of the Earth. The positive feedback mechanism in the tracking mode will maintain the resonance of the oscillatory systems, ensuring the process of advanced deformation of the object's structures. What can be interpreted as the unsupported motion of one object in the interference field of the spatial frequencies of another object, or according to the accepted terminology, this type of motion in the usual sense can be called movement under the action of "gravitational forces".

The formation of its own structure of spatial frequencies by placing a system of parametric emitters of longitudinal waves on the object makes it possible to transfer the type of interaction from passive to active and thereby compensate for the reactive component, that is, to compensate for the force of gravity. Simultaneously, using the same emitters, one can control the state of the interference structure of the external field of the object and, by changing the direction of the resulting vector, change the direction of the object's movement. Thus, the interference structure created by the phased array interacts with the natural structure of the geometric field of spatial frequencies, that is, interacts directly with the holographic wave matrix. In other words, by changing the structure of the wave matrix at the programmatic level of physical reality, we thereby change the materialized projection of this matrix in the form of a force field at the object level of physical reality.

\section{Conclusions}

The considered physical principle, based on the interaction of the natural structure of spatial frequencies in the form of the earth's frequency funnel, with a certain way created asymmetric interference structure of longitudinal waves generated by the propulsion system, makes it possible to develop a model of a platform for unsupported movement in space. Thus, confirming the existence of a huge technological potential inherent in the new interpretation of the structure of space.

\section{References}

[1]. Igor Maydykovskiy, Petras Užpelkis: The Physical Essence of Time // Intellectual Archive, 2020, pp. 16. https://doi.org/10.32370/IAJ.2450

[2]. Igor Maydykovskiy, Petras Užpelkis: Holographic principle as the Basis for the Existence of Physical Reality // Intellectual Archive, 2020, pp. 16. https://doi.org/10.32370/IAJ.2348

[3]. Murray and Dermott: Solar System Dynamics // Cambridge University Press 1999. ISBN 0-521$\underline{57597-4}$

[4]. R. V. Pound, Jr. G. A. Rebka: Apparent weight of photons // Physical Review Letters, Journal. 1960. 1 April (vol. 4, no. 7) - P. 337-341. https://journals.aps.org/prl/pdf/10.1103/PhysRevLett.4.337

[5]. Igor Maydykovskiy, Petras Užpelkis: Sagnac effect - experimental confirmation of the Theory of Phase Medium. // Intellectual Archive, 2019, pp. 12. https://doi.org/10.32370/IAJ.2204 